\title{
Neurological complications of bariatric surgery
}

Hussein A. Algahtani, MD, FRCPC, AbidS. Khan, $M R C P(U K)$, MuhammadA. Khan, MBBS, MBA, AhmedA. Aldarmahi, MME, $P h D$, YousifLodhi, Medical Student.

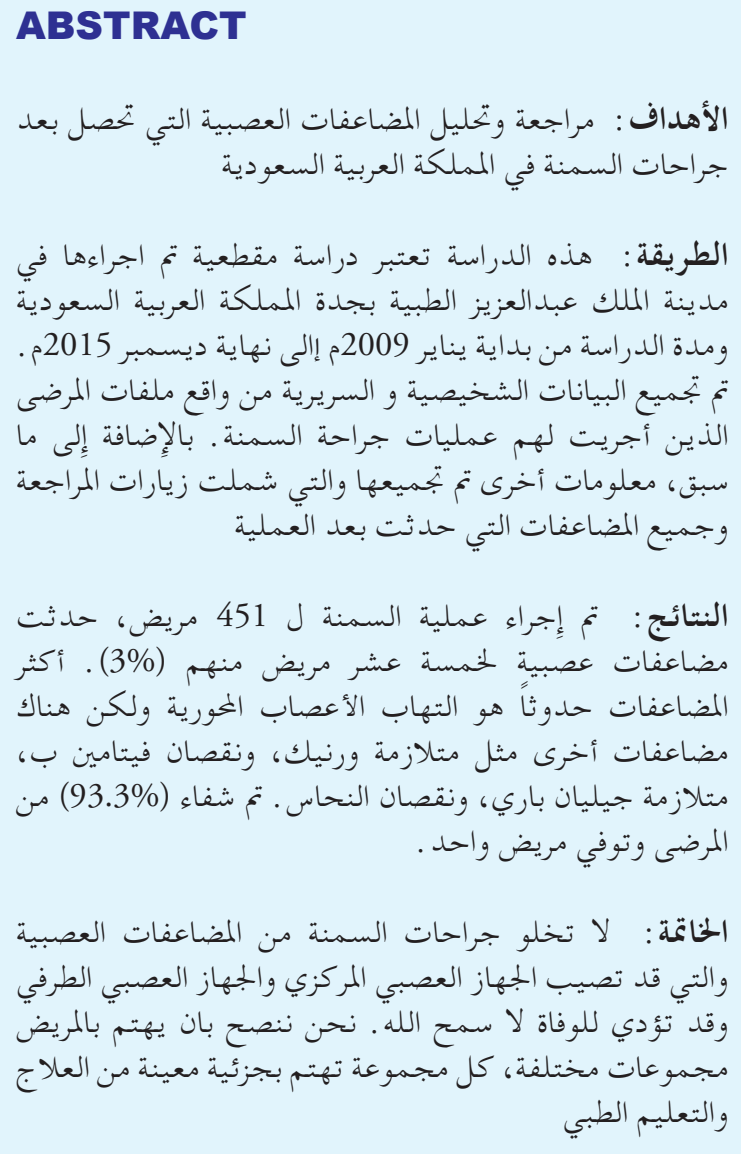

Objective: To review and analyze the neurological complications from bariatric surgery in Kingdom of Saudi Arabia.

Methods: This cross sectional study was carried out in King Abdulaziz Medical City, Jeddah, Kingdom of Saudi Arabia from January 2009 to December 2015. Important personal and clinical data were collected from the charts of the patients who underwent bariatric surgery. Data on follow up visit and remote complication if present, was also collected. All patients with neurological complications were reviewed in detail. The significant difference was calculated by using T-test and $p$-value $<0.05$ was considered significant.

Results: A total of 451 patients underwent bariatric surgery, 15 cases had neurological complications (3\%). Axonal polyneuropathy was the most frequent neurological complication, but cases of Wernicke syndrome, vitamin B12 deficiency, Guillain-Barre syndrome and copper deficiency were also identified. Fourteen patients $(93.3 \%)$ had full recovery from the neurological signs and symptoms; one patient died.

Conclusions: Bariatric surgery is not free of potential neurological complications. Complications may affect both central and peripheral nervous system and death is a possibility. Multidisciplinary care including consultation of different teams is highly recommended.

Neurosciences 2016; Vol. 21 (3): 241-245 doi: 10.17712/nsj.2016.3.20160039

From the College of Medicine (Algahtani, Khan M, Aldarmahi), King Saud Bin Abdulaziz University for Health Sciences, the Neurology Section (Khan A), King Abdulaziz Medical City, and the College of Medicine (Lodhi), Jeddah, Kingdom of Saudi Arabia.

Received 24th January 2016. Accepted 9th March 2016.

Address correspondence and reprint request to: Dr. Hussein A. Algahtani, College of Medicine, King Saud bin Abdulaziz University for Health Sciences, Jeddah, Kingdom of Saudi Arabia. Tel. +966 (21) 6240000 Ext 21298/22070.

E-mail:halgahtani@hotmail.com

verweight refers to a weight above the "normal" range, with normal defined on the basis of actuarial data. ${ }^{1}$ This is determined by calculating the body mass index (BMI) defined as the weight in kilograms divided by height in meters squared. Overweight is defined as a BMI of 25 to $29.9 \mathrm{~kg} / \mathrm{m}^{2}$, and obesity is defined as a BMI of $\geq 30 \mathrm{~kg} / \mathrm{m}^{2}$. Severe obesity is defined as a BMI $\geq 40$ $\mathrm{kg} / \mathrm{m}^{2}$ (or $\geq 35 \mathrm{~kg} / \mathrm{m}^{2}$ in the presence of co-morbidities). Obesity is reaching pandemic proportion with more than $26 \%$ of American adults and children obese. ${ }^{1}$ An 
additional $35 \%$ of the American adult population is overweight, with a BMI of $25 \mathrm{~kg} / \mathrm{m}^{2}$. In Saudi Arabia, data is even more striking; a total of $72.5 \%$ of Saudis are either overweight or obese. ${ }^{2}$ The annual mortality attributed to this condition in the United States of America (USA) is estimated to be around 280,000 to 300,000 deaths. $^{3}$ Due to the pandemic nature of obesity and limited efficacy of the available medical treatment, there has been a steady increase in utilization of bariatric surgical procedures (BSP), especially for medically complicated obesity. The current estimated number of BSP carried out annually in the USA is approximately 350,000 and the total annual cost of obesity is more than 147 billion dollars. ${ }^{4} \mathrm{~A}$ variety of neurological complications of BSP are well documented in the literature. Neurological complications range from $5-16 \%$ of the patients. ${ }^{5}$ To date, there is a lack of studies on neurological complications of BSP conducted in Saudi Arabia. The aim of this study was to review the neurological complications of BSP and analyze the possible causes for their occurrence.

Methods. This was a cross sectional study carried out in the Neurology Unit, King Abdulaziz Medical City, Jeddah, Kingdom of Saudi Arabia. The medical city is a tertiary hospital with a capacity of 650 beds, which provides comprehensive services in neurology, and engages in both undergraduate and postgraduate training and research. In this study, we reviewed all charts of the patients who underwent BSP from January 2009 to December 2015. The total number of patients who were included was 451 . Variables collected were age, gender, BMI, co-morbidities, type of procedure, and duration of stay in hospital, complications, and postoperative follow up. In addition, remote complications if present were also documented. All patients with neurological complications were reviewed including their clinical examination, laboratory and imaging results, treatment, and prognosis. This study was approved by King Abdullah International Medical Research Center. Patient confidentiality was assured by identification of only file number without names or photos. The sample enrolled in this study was all patients admitted and operated on at King Abdulaziz Medical City, Jeddah, Kingdom of Saudi Arabia for BSP with no patient excluded. Furthermore, patients with neurological complications were analyzed including clinical and paraclinical data.

All data were entered and analyzed using Statistical Package for Social Silences, version 21 (SPSS Inc, Chicago, IL, USA). Descriptive statistics was calculated and include percentages, means, and standard deviations
(SD). The significant difference was calculated by using t-test. The significance for $p$-value was $<0.05$.

Results. The total number of patients who were included in our study was 451. A total of 140 patients were males (31\%) and 311 were females (69\%). The mean age was $36.7 \pm 11.7$ for males and $37.5 \pm 10.8$ for females (Table 1). According to BMI, $71 \%$ of our patients were morbidly obese (BMI >40), 26\% severely obese $(\mathrm{BMI}>35)$ and $3 \%$ were obese (BMI 30-35). None of the patients were overweight (BMI 25-30) or had normal BMI who underwent BSP. The mean BMI was $48.1 \pm 8.2$ for males, and $44.1 \pm 7.7$ for females. Differences in gender were significant for BMI categories and surgical procedures. More males were in the morbidly obese category $(86.4 \%)$ and severely obese $(13.6 \%)$ as compared with females who were scattered throughout obesity scale. Females' obesity distribution was as follows: obese (4.2\%), severely obese (31.2\%) and morbidly obese $(64.6 \%)$, with $p$-value of $<0.001$. The number of patients with BMI $>35$ without a co-morbid condition who had BSP was 13 (3\%).

The most common surgical procedure performed was laparoscopic sleeve gastrectomy $(88 \%)$, followed by Roux-en-y gastric bypass (10.2\%), and gastric banding (1.8\%) (Table 2 ). The type of surgical procedure opted

Table 1- Description of clinical characteristics of patients undergoing bariatric surgery for obesity.

\begin{tabular}{|c|c|c|}
\hline \multirow{2}{*}{ Variables } & Males & Females \\
\hline & \multicolumn{2}{|c|}{ Mean (SD) } \\
\hline Age (years) & $36.7( \pm 11.7)$ & $37.5( \pm 10.8)$ \\
\hline BMI $\left(\mathrm{kg} / \mathrm{m}^{2}\right)$ & $48.1( \pm 8.2)$ & $44.1( \pm 7.7)$ \\
\hline Hospital stay (days) & $2.2( \pm 1.5)$ & $2.0( \pm 1.0)$ \\
\hline Follow up (years) & $2.7( \pm 1.0)$ & $2.7( \pm 1.0)$ \\
\hline
\end{tabular}

Table 2 - Differences between gender according to surgical procedures and BMI categories among bariatric surgery patients. $(\mathrm{N}=451)$

\begin{tabular}{|c|c|c|c|}
\hline \multirow{3}{*}{ BMI Categories } & \multicolumn{2}{|c|}{ Gender } & \multirow{3}{*}{$P$-value } \\
\hline & Male $n=140$ & Females $\mathbf{n}=311$ & \\
\hline & \multicolumn{2}{|c|}{$\mathbf{n}(\%)$} & \\
\hline Obese & $0(0)$ & $13(4.2)$ & \\
\hline Severely obese & $19(13.6)$ & $97(31.2)$ & $<0.001^{*}$ \\
\hline Morbidly obese & $121(86.4)$ & $201(64.6)$ & \\
\hline \multicolumn{4}{|l|}{ Surgical procedures } \\
\hline Roux-en-Y gastric bypass & $26(18.6)$ & $20(6.4)$ & \multirow{3}{*}{$<0.001^{\prime}$} \\
\hline $\begin{array}{l}\text { Laparoscopic sleeve } \\
\text { gastrectomy }\end{array}$ & $110(78.6)$ & $287(92.3)$ & \\
\hline Gastric banding & $4(2.9)$ & $4(1.3)$ & \\
\hline \multicolumn{4}{|c|}{ *Fisher-exact Test, BMI - body mass index } \\
\hline
\end{tabular}


by the patient was different between males and females despite being given choices among different surgical procedures. Males preferred Roux-en-y-gastric bypass, while females preferred laparoscopic sleeve gastrectomy $p$-value $=0.001$. Pre-operatively, all patients had routine blood work, electrocardiography, and chest radiographs. In addition, other tests were ordered based on the assessment of the pre-operative assessment clinic.

The percentage of patients who developed neurological complications (3\%) is lower than those reported in the international literature (4.6 to 16\%). Out of the 15 patients with complications, 9 developed peripheral neuropathy, Guillain-Barre syndrome (2), vitamin B12 deficiency (2), copper deficiency (1), and Wernicke encephalopathy (1). A summary of these cases is shown in Table 3. Diagnosis of peripheral neuropathy (9 patients) was confirmed by both clinical features and neurophysiological studies. The work up for an underlying cause was unremarkable, and think it is most likely due to nutritional deficiency. One case of Wernicke encephalopathy was diagnosed (Figure 1). She had recurrent nausea and vomiting and fortunately she responded to intravenous thiamin replacement therapy. The time elapsed between the surgical procedure and

Table 3 - Summary of neurological complications in our study among bariatric surgery patients.

\begin{tabular}{lll}
\hline Comorbid condition & $\mathbf{n}$ & $(\%)$ \\
\hline Peripheral neuropathy & 9 & $(2)$ \\
Vitamin B12 deficiency & 2 & $(0.4)$ \\
Guillain-Barre syndrome & 2 & $(0.4)$ \\
Wernicke encephalopathy & 1 & $(0.2)$ \\
Copper deficiency & 1 & $(0.2)$ \\
No neurological complications & 436 & $(96.7)$ \\
Total & $\mathbf{4 5 1}$ & $\mathbf{( 1 0 0 )}$ \\
\hline
\end{tabular}

the onset of neurological manifestation ranged from 10 days to 2 years. Although compression neuropathies caused by rapid weight loss and the habit of adjusting to wrong postures while sitting (for example crossing the legs) or sleeping are well-described complications, no cases of compression neuropathy were recorded in our study. In addition, no cases of plexopathies, optic nerve or white matter diseases were identified. All patients with neurological complications recovered completely except one patient with Guillian-Barre syndrome who developed severe depression and subsequently died from massive pulmonary embolism. The duration of hospital stay in days was $2.2 \pm 1.5$ for males and $2.0 \pm 1.0$ for females. The mean follow up (years) for all patients was $2.7 \pm 1.0$. Post-operative follow up was as follows: one visit (16.2\%), 2 visits (21.5\%), and 3 visits (34.6\%). Unfortunately $27.7 \%$ of patients did not attend the clinic after BSP.

The most common comorbid conditions were hypertension, diabetes mellitus, dyslipidemia, and obstructive sleep apnea (Table 4). We noticed improved diabetic control in $5 \%$ of patients and ultimately $2 \%$ of

Table 4 - List of the common co-morbidities present in our study among bariatric surgery patients.

\begin{tabular}{llr}
\hline Comorbid condition & $\mathbf{n}$ & $(\%)$ \\
\hline Hypertension & 87 & $(19.3)$ \\
Diabetes mellitus & 68 & $(15.1)$ \\
Dyslipidemia & 43 & $(9.5)$ \\
Obstructive sleep apnea & 33 & $(7.5)$ \\
Hypothyroidism & 33 & $(7.3)$ \\
Asthma & 25 & $(5.5)$ \\
Joint disease & 23 & $(5.3)$ \\
Gastroesophageal reflux disease & 8 & $(1.8)$ \\
Idiopathic intracranial hypertension & 2 & $(0.4)$ \\
Back pain & 1 & $(0.2)$ \\
\hline
\end{tabular}
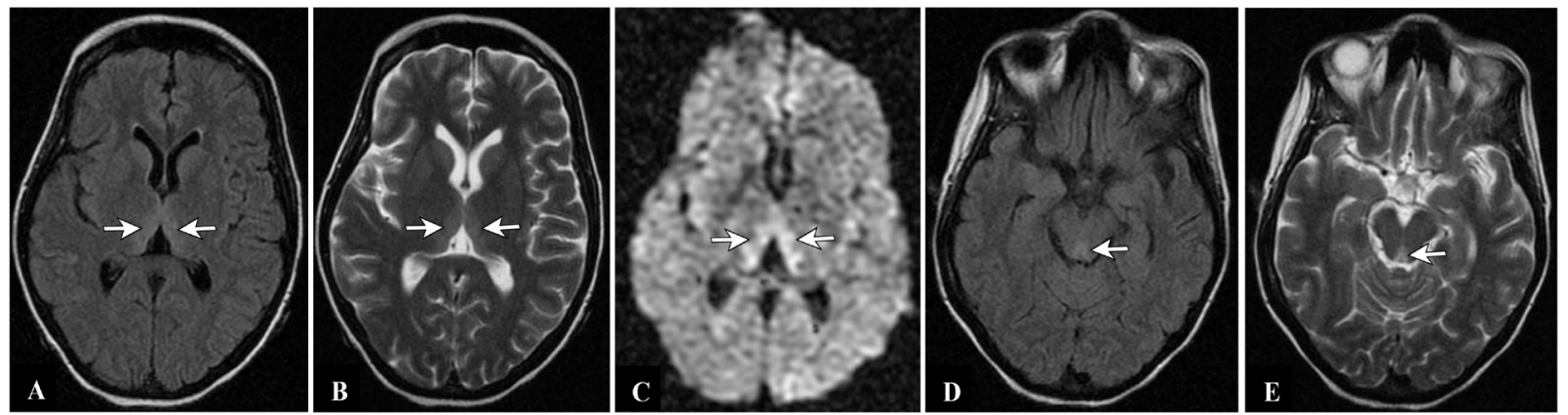

Figure 1 - Magnetic resonance imaging brain of one of our patients who developed Wernicke's encephalopathy following BSP. Images shown are axial images at the level of thalami (A, B, and C) and midbrain (D and E). Sequences are FLAIR images (A and D), T2 WI (B and E) and diffusion image (C). Images show high signal intensity in the medial thalamic nuclei and tectal plate. BSP- bariatric surgical procedures, FLAIR - fluidattenuated inversion recovery 


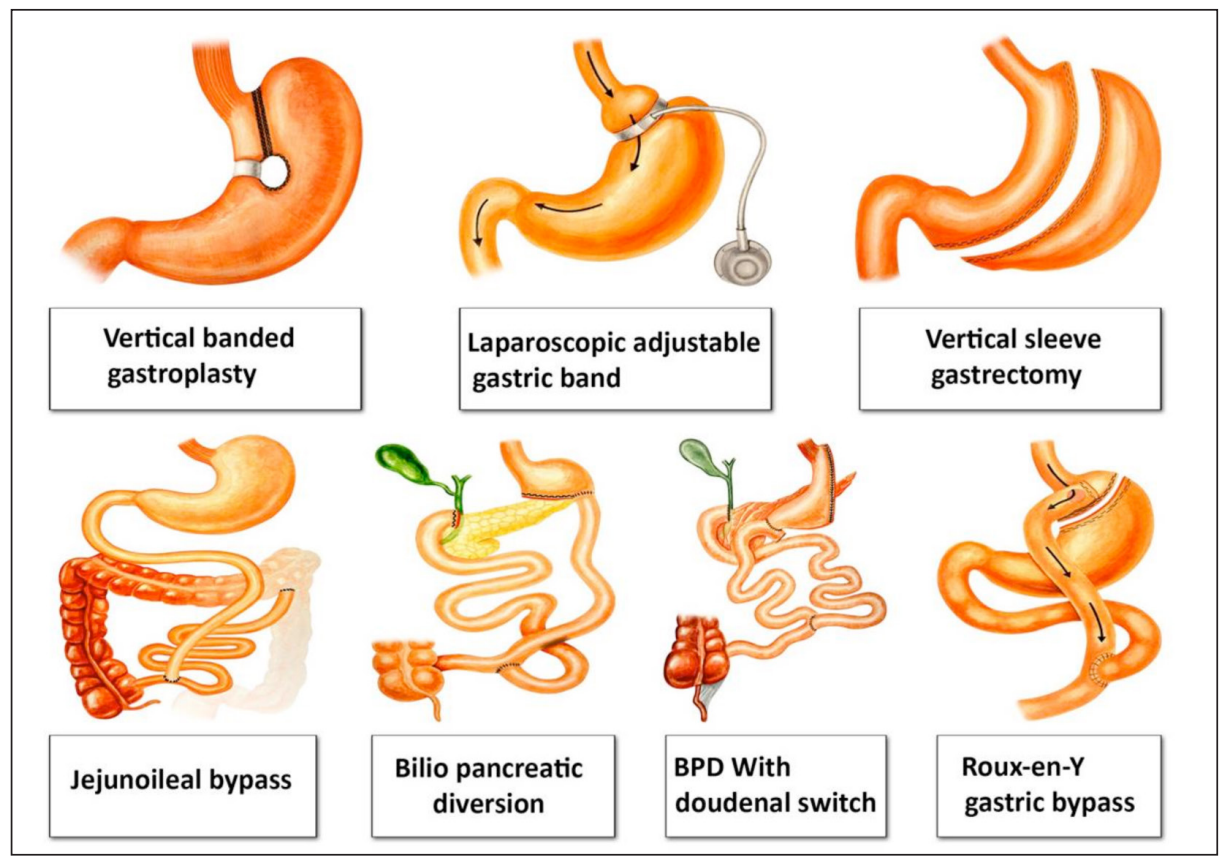

Figure 2 - Simple drawing of different types of BSP. BSP - bariatric surgical procedures, BPD - biliopancreatic diversion

patients stopped insulin and oral hypoglycemic therapy. In addition, lipid control has improved in $2 \%$ of patients. Losing weight did not help in the management of hypothyroidism, however, the dose of thyroxine had to be adjusted. Interestingly, 2 BSPs were carried out to treat idiopathic intracranial hypertension $(\mathrm{IIH})$ with improvement in their disease. Their fundi examination was normal after the procedure, and treatment for $\mathrm{IIH}$ was stopped.

Discussion. Bariatric surgeries are becoming a popular procedures widely disseminated in the world. A number of BSP have been employed for treating obesity and achieving weight reduction (Figure 2). Early BSP fell out of favor because of significant weight loss and malnutrition. These included jejunoileal bypass, duodenal switch and biliopancreatic diversion with duodenal switch. Roux-en-Y gastric bypass and gastroplasties including sleeve gastrectomy and vertical gastric banding are the most common BSP performed in the USA. Many of these BSP can be performed either endoscopically or laparoscopically. Vertical banded gastroplasty has high rates of suture line failures and gastrogastric fistulas. Roux-en-Y is invasive and laparoscopic adjustable gastric banding does not achieve the weight loss desired. This makes vertical sleeve gastroplasty the procedure of choice nowadays. Intragastric balloons and endoscopially placed devices are being tried or studied and are only available outside the United States.

Although guidelines and inclusion criteria govern the selection and follow up of patients, these are not strictly followed. ${ }^{7}$ Patients think that bariatric surgery is the solution for their frustration and a suitable alternative to a balanced diet and drastic changes in lifestyle. A Google search yielded more than 8 million hits for "bariatric surgery", and around 70000 for "private clinics bariatric surgery". In our study, the number of BSP was noticed to be exponentially increasing over recent years. Rapid and significant weight loss may be associated with deficiency of important vitamins and minerals. While reviewing the literature, it was noticed that patients who developed neurological complications related to nutritional deficiencies have a history of repeated attacks of vomiting, lack of poor dietary intake, or lack of vitamin and mineral supplement, ${ }^{6,7}$ and this was also observed in our study. Repeated vomiting should be treated promptly to avoid such complications. Our patients were not followed by a dietician on a regular basis, a practice that should be changed. We have noticed during follow up and despite late presentation and genuine neurological deficit, full recovery and good outcome of patients with nutritional deficiencies following appropriate supplementation. Nutritional deficiencies were replenished either orally, by intravenous route like thiamine (B1) or by the intramuscular route like cyanocobalamin (B12). There were more cases of 
nutritional deficiency-related peripheral neuropathy. These patients presented with burning feet and hand syndrome. Diagnosis was confirmed both by clinical features, nerve conduction, and electromyography tests. These patients improved on nutritional supplement and symptomatic treatment. One patient developed severe selenium deficiency and diagnosis was confirmed by typical clinical presentation and serum selenium levels. She presented with white matter disease and features suggestive of multiple sclerosis like syndrome. This patient improved after selenium supplement, however, it took her 3 months to recover fully. In our study, all patients had severe vitamin D deficiency. Vitamin D deficiency is quite common in Saudi Arabia, ${ }^{8}$ with a prevalence that ranges from $40-95 \%$. We recommend monitoring and full supplement of vitamin D and calcium to all patients undergoing bariatric surgery.

We have also noticed an improvement in co-morbid conditions following bariatric surgery. These included diabetic control of blood glucose (reduce/eliminate the need for insulin and oral hypoglycemic agents), hypertension, degenerative joint disease, IIH, and obstructive sleep apnea. The complications of bariatric surgery are not insignificant. ${ }^{9}$ In one series, major complications occurred in $3.3 \%$ of patients, and the in-hospital mortality was $0.4 \%$. Neurological complications reported in the literature include polyneuropathy, mononeuropathy, Guillain-Barre syndrome, Wernicke's encephalopathy, ataxia and myopathy. In our study, one patient died and the mortality rate is not different from other centers reports $(0.2 \%)$. Unfortunately, none of the patients were informed on the possible neurological complications. In fact, patients regarded the BSP as trivial and simple. We can blame on media coverage of celebrities undergoing these procedures in order to lose weight quickly.

We are aware of the limitations of this study (cross sectional), however, most studies published on this subject follow the same methodology. In addition, the presented review of literature sheds light on the condition world-wide. Another limitation is lack of proper follow up of patients $(27.7 \%)$. The reason behind this is not clear to us; however, we can speculate that among the reasons for not attending the clinics after BSP was lack of proper awareness/education before surgery and lack of an organized bariatric multidisciplinary team that should have contacted the patient/family. Other factors include eligibility, financial issues, and ease of accessibility to other hospitals.

In conclusion, bariatric surgery, a procedure that is continuously increasing in popularity, is not free of potential neurological complications. Neurological complications range from $5-16 \%$ of the patients. The adverse effect of each BSP should be clearly explained to the individual undergoing this type of surgery. Although a clear cause-effect relation cannot be established for the present cases, the cumulative literature on the subject makes it important to warn the patients of the potential risks. A clear awareness program educating the patients should be held regularly; guidelines and follow-up schedules should be planned and practiced including operating surgeon, dietician, and family physician.

Acknowledgment. The authors highly appreciate the contribution of Mr. Ashraf Algadhi for his hand made drawing (Figure 2).

\section{References}

1. Berger JR. The neurological complications of bariatric surgery. Arch Neurol 2004; 61: 1185-1189.

2. Berger G. Obesity and managed care. Manag Care Interface 2007; 20 : 36-37.

3. Al-Nuaim AA, Bamgboye EA, al-Rubeaan KA, al-Mazrou Y. Overweight and obesity in Saudi Arabian adult population, role of socio-demographic variables. J Community Health 1997; 22: 211-223.

4. Kumar N. Obesity surgery: a word of neurologic caution. Neurology 2007; 68(21): E36-E38.

5. Abarbanel JM, Berginer VM, Osimani A, Solomon H, Charuzi I. Neurologic complications after gastric restriction surgery for morbid obesity. Neurology 1987; 37: 196-200.

6. Juhasz-Pocsine K, Rudnicki SA, Archer RL, Harik SI. Neurologic complications of gastric bypass surgery for morbid obesity. Neurology 2007; 68: 1843-1850.

7. Thaisetthawatkul P, Collazo-Clavell ML, Sarr MG, Norell JE, Dyck PJ. A controlled study of peripheral neuropathy after bariatric surgery. Neurology 2004; 63: 1462-1470.

8. Al-Mogbel ES. Vitamin D status among Adult Saudi Females visiting Primary Health Care Clinics. Int J Health Sci (Qassim) 2012; 6: 116-126.

9. Ba F, Siddiqi ZA. Neurologic complications of bariatric surgery. Rev Neurol Dis 2010; 7: 119-124. 